\title{
Investigation of Water Uptake in Porous Asphalt Concrete Using Neutron Radiography
}

\section{Journal Article}

\section{Author(s):}

Lal, Sreeyuth; Poulikakos, Lily D.; Sedighi Gilani, Marjan; Jerjen, Iwan; Vontobel, Peter; Partl, Manfred N.; Carmeliet, Jan C.; Derome, Dominique

\section{Publication date:}

2014

\section{Permanent link:}

https://doi.org/10.3929/ethz-b-000089582

\section{Rights / license:}

In Copyright - Non-Commercial Use Permitted

Originally published in:

Transport in Porous Media 105(2), https://doi.org/10.1007/s11242-014-0376-6 


\title{
Investigation of Water Uptake in Porous Asphalt Concrete Using Neutron Radiography
}

\author{
Sreeyuth Lal · Lily D. Poulikakos • Marjan Sedighi Gilani • \\ Iwan Jerjen · Peter Vontobel • Manfred N. Partl • \\ Jan C. Carmeliet • Dominique Derome
}

Received: 3 March 2014 / Accepted: 6 August 2014 / Published online: 29 August 2014

C) Springer Science+Business Media Dordrecht 2014

\begin{abstract}
Porous asphalt (PA), a highly porous hydrophobic composite material, is subjected to water uptake and the process is documented with neutron radiography (NR). While the un-aged laboratory-prepared PA specimens do not show any water uptake, we observe uptake in aged PA even though the bitumen binder is a hydrophobic material. The moisture content distribution plots derived from the NR images clearly identify regions in the aged specimens where water uptake is active. Two-dimensional degree of saturation (DoS) distribution images, which are obtained by combining micro-computer tomography and NR
\end{abstract}

S. Lal $(\varangle)$. J. C. Carmeliet · D. Derome

Laboratory for Building Science and Technology, Empa, Swiss Federal Laboratories for Materials Science and Technology, Überlandstrasse 129, 8600 Dübendorf, Switzerland

e-mail: Sreeyuth.1al@empa.ch

S. Lal

Department of Civil, Environmental and Geomatic Engineering, ETH Zurich, 8093 Zurich, Switzerland

L. D. Poulikakos · M. N. Partl

Laboratory for Road Engineering/Sealing Components, Empa, Swiss Federal Laboratories for Materials Science and Technology, Überlandstrasse 129, 8600 Dübendorf, Switzerland

M. Sedighi Gilani

Laboratory for Applied Wood Materials, Empa, Swiss Federal Laboratories for Materials Science and Technology, Uberlandstrasse 129, 8600 Dübendorf, Switzerland

I. Jerjen

Centre for X-ray Analytics, Empa, Swiss Federal Laboratories for Materials Science and Technology, Uberlandstrasse 129, 8600 Dübendorf, Switzerland

P. Vontobel

Spallation Neutron Source Division ASQ, Paul Scherrer Institut, 5232 Villigen, PSI, Switzerland

M. N. Partl

Division of Highway and Railway Engineering, KTH Stockholm, 10044 Stockholm, Sweden

J. C. Carmeliet

Chair of Building Physics, ETH Zürich, 8093 Zurich, Switzerland 
images, identify those pores where saturated flow is certainly active. However, to clearly distinguish between saturated and unsaturated flows in the remaining wet pores, the DoS distribution images are read together with the three-dimensional PA microstructure obtained by micro-CT. It is observed that uptake begins mainly as unsaturated film/corner flow at large well-defined pores. As this uptaken water travels further into the material, the flow transforms into a combination of saturated flow and unsaturated film/corner flow. Saturated flow is seen to be mostly active in the small pores within the mastic. From the observed succession of unsaturated and saturated flows in an aged PA specimen, it can be concluded that years of environmental and mechanical loading has resulted in the stripping of binder from the aggregate surfaces and has consequently exposed patches of hydrophilic aggregate to water, which enables the capillary uptake of water. We also simulate an absolute permeability experiment and observe that relatively less tortuous and more connected paths play an important role in determining the preferential path of the uptaken water.

Keywords Water uptake - Porous asphalt · Neutron radiography · Saturated flow · Unsaturated flow

\section{Introduction}

Porous asphalt (PA) is a material used as the surface layer of roads to allow the drainage of water and thereby prevent aquaplaning and splash-spray effects during rain events. In combination with a well-designed impervious asphalt concrete layer, which acts as a structural support for the upper PA layer, PA has proven to be an efficient tool in water-management strategies of many cities (Cahill et al. 2004). The acoustical absorption property of PA leads to a significant absorption of both engine and tire noises (Meiarashi et al. 1996). The good hygric and acoustic properties are used to promote PA as an environment-friendly road material. PA typically has a porosity of around $20 \%$ and is a composite material made from fine and coarse mineral aggregates, a bituminous binder and air voids. In some cases, like in one of the specimens in this study, fibers are also added to PA to allow thick binder films for the sake of improved mechanical behavior and reduced binder drain-down effects. The microstructure analysis of PA (Fig. 1) shows the components in a typical PA internal structure. Ideally, the bituminous binder covers all particles with a thin hydrophobic layer. A description of a typical microstructure of PA is given in Poulikakos and Partl (2010).

Due to its high porosity, the internal structure of PA is exposed to water. Hence, PA is more susceptible to moisture-induced deterioration than dense asphalt concrete, resulting in a shortened service life. Water induces deterioration in PA primarily by affecting binder cohesion and the adhesion between binders and aggregates (Partl et al. 2008; Kim et al. 2009; Poulikakos and Partl 2012). In recent papers, Poulikakos et al. (2013a, b) analyzed water drainage and drying from PA using neutron radiography (NR). The complexity of gravity-driven drainage pattern of water within PA was elucidated. It was also observed that, although hydrophobic, PA retained water after a drainage event and that $8-10 \mathrm{~h}$ of convective air flow above the specimen was not sufficient to completely dry it.

In the past, researchers have effectively used neutron imaging techniques in porous media research. The measurements have ranged from one- and two-dimensional moisture propagation analysis (e.g., El Abd and Milczarek 2004; Cnudde et al. 2008) to three-dimensional structure analysis with neutron tomography (e.g., Satija et al. 2004). A good review of the advances in NR is given by Strobl et al. (2009). However, as compared to microporous media, PA displays a very different pore size distribution and pore structure, where 1D simplification 
(a)

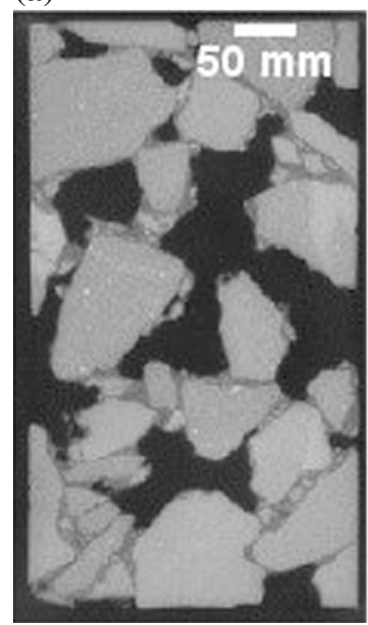

(b)

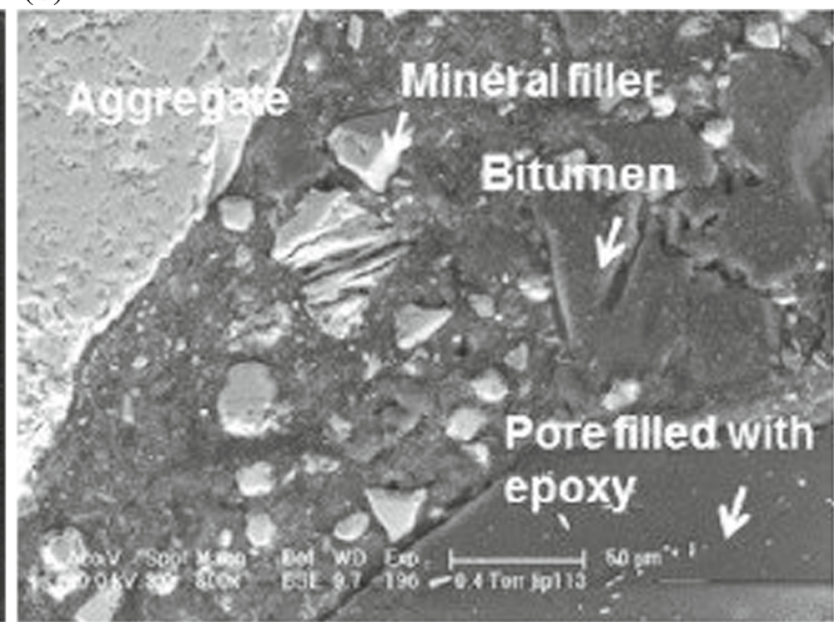

Fig. 1 Microstructure of porous asphalt concrete consisting of coarse and fine mineral aggregates, bitumen and air voids: a microtomographic slice of the specimen, where voids are black, aggregates light gray and bitumen darker gray, and b ESEM micrograph, the voids are filled with epoxy resin (scale bar $50 \mu \mathrm{m}$ ) (Figure adapted from Poulikakos et al. 2013a)

of moisture propagation cannot be made. Moreover, though laboratory tests can be used to study unidirectional permeability of water in asphalt, field testing measures a combination of horizontal and vertical permeability (Maupin 2001). Also, one-dimensional standardized permeability methods are not suitable for a fundamental understanding on a micromechanicalphysical level. Hence, two-dimensional NR can be a very effective method to track water propagation in PA, especially if it is used complementarily with field permeability testing.

In the aftermath of a large rain event, and eventual sitting water pools, there is a possibility of water uptake in the top PA layer. Unlike drainage, in which the contribution of larger pores to water propagation is dominant, in uptake, smaller pores also play a role. In effect, whereas gravity is the driving force behind drainage, capillary forces due to surface tension effects drive water uptake. At low saturation, flow in the form of thin liquid films also becomes an important mode of moisture propagation (Tuller and Or 2001). In particular, the question of water redistribution due to capillary effect arose from previous works (Poulikakos et al. 2013a, b).

The objective of this study is to understand the phenomenon of steady water uptake in $\mathrm{PA}$ at room temperature by combining NR and microtomography imaging. To investigate this behavior, in this work, we place PA in contact with a free pool of water and document liquid uptake in different specimens. By combining NR images with pore structure information obtained from microtomographic scans, we aim to distinguish between the saturated and unsaturated flows within the PA structure during water uptake. In addition, using finite volume computer simulations, we assess the influence of tortuosity and pore connectivity in determining the rate of vertical water uptake.

In the next section, first the characteristics of the three specimens investigated are detailed, followed by a brief description of the imaging techniques used and the details of the experimental procedure. We then present the results, discuss them in detail, list future avenues of research, and finally present our conclusions. 
Table 1 Material specifications

\begin{tabular}{lll}
\hline & AG1 $^{\mathrm{a}}$ & VD4 $^{\mathrm{b}}$ \\
\hline EN 13108-7 designation & PA11 & PA11 \\
\hline Voids (\% by vol) & 21 & 22.8 \\
\hline Sieve size (mm) & Percent passing & 100 \\
16 & 100 & 95 \\
11.2 & 98 & 49 \\
8 & 60 & 20 \\
5.6 & 16 & 15 \\
4 & 13 & 13 \\
2.8 & 12 & 11 \\
2 & 11 & 7 \\
1 & 8 & 6 \\
0.5 & 7 & 4.8 \\
0.09 & 4.8 & PmB $^{\mathrm{c}}$ Styrelf \\
Penetration-Graded $13 / 80$ \\
Binder (weight- $\%$ of total mixture) & Bit.55/70 +Trinidad NAF 501 & 4.8 \\
Penetration at $25^{\circ} \mathrm{C}(0.1$ mm) & 4.8 & 50 \\
Softening point $\left({ }^{\circ} \mathrm{C}\right)$ & 78.2 & 56.6 \\
Aggregate type & Siliceous limestone & Quartz sandstone \\
Los Angeles abrasion test (LA) & $13-18$ & $<18$ \\
Polished stone value test (PSV) & 53 & $>50$ \\
\hline
\end{tabular}

a Laboratory-produced specimen

b Field core

c Polymer-modified bitumen

\section{Materials and Methods}

\subsection{Materials}

Two different PA materials with known in situ performance (Poulikakos et al. 2006) were used in this study for comparison purposes. These mixtures were used in the Swiss cantons Aargau (referred to as AG1) and Vaud (referred to as VD4). VD4 is part of a large piece that was harvested from a road after 7 years of service. AG1 was freshly made in the laboratory using the mix design specifications of the road that had been monitored for 5 years. The composition of these two materials differ firstly in the aggregate gradation, with VD4 having a slightly higher proportion of aggregates in the $8-11.2 \mathrm{~mm}$ range than in the $5.6-8 \mathrm{~mm}$ range as compared to AG1, and secondly in the type and content of the bituminous binder (Table 1). VD4 contains polymer-modified bitumen, with styrene butadiene styrene (SBS) co-polymer. AG1 has 55/70 penetration-graded straight run bitumen with Trinidad natural asphalt with NAF 501 additive; a fine-grained granular product, consisting of 83.3 weight$\%$ Trinidad Epuré and 16.7 weight-\% cellulose fibers (http://www.trinidad-lake-asphalt.de/ english/Produkte-NAF-501.php). Using the sessile drop method, the static contact angle of a drop of water was found to be more than $90^{\circ}$ in both binders (Poulikakos and Partl 2012) i.e., both of them can be categorized as hydrophobic. The mineral aggregate of AG1 is siliceous 


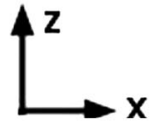

(a)

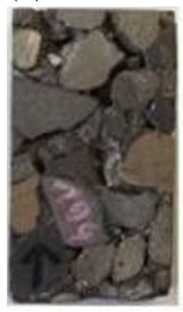

(f)

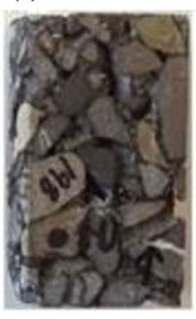

(b)

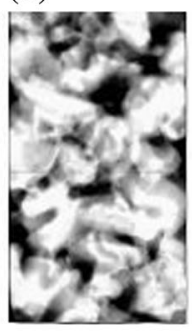

(g)

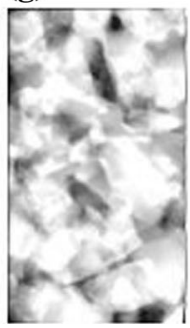

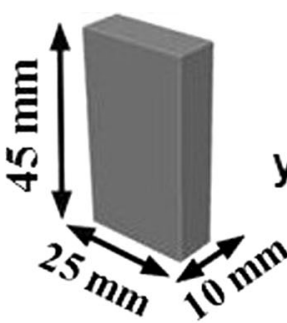

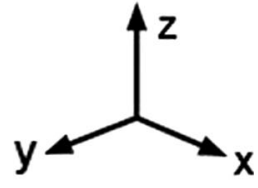

VD4-1

(c)

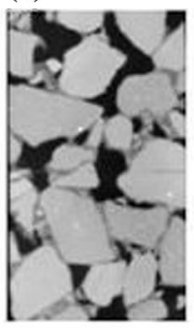

(d)

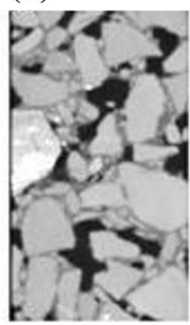

(e)

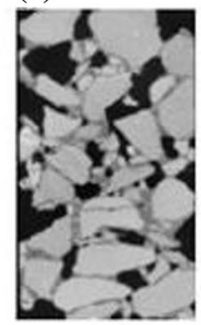

(h)
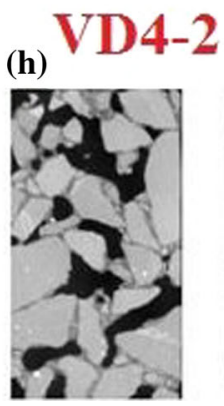

(i)

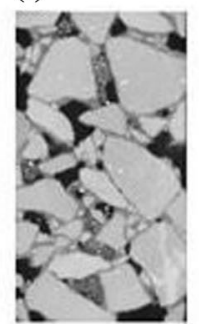

(j)

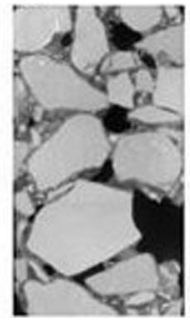

Fig. 2 Visible-light photographs $(\mathbf{a}, \mathbf{f}), 2 \mathrm{D}$ cumulative porosity distribution images $(\mathbf{b}, \mathbf{g})$ in which the porosity ranges from 0 (white) to 1 (black) and microtomographic slices at the front $(\mathbf{c}, \mathbf{h})$, middle $(\mathbf{d}, \mathbf{i})$ and rear $(\mathbf{e}, \mathbf{j})$ parts of VD4-1 and VD4-2

limestone from the Balmholz quarry with high pressure and abrasion resistance as well as good water resistance. Mineralogical data indicate 30-45\% quartz by mass (www.balmholz. ch). The mineral aggregates used in VD4 are from the Famsa quarry, a hard sand stone from the sedimentary rock category with 20-30 \% quartz (www.famsa.ch). Three specimens are used in this study, one from the AG1 pavement (named AG1) and two specimens from the VD4 pavement (named VD4-1 and VD4-2). The two specimens from VD4 are simply two parts of the same material. The specimens have been chosen in such a way that it enables us to study the effect of both spatial inhomogeneity (two specimens from the same material) as well as different chemical and physical properties (specimens from laboratory-made and in situ materials) on the water uptake process. All three specimens selected have the dimensions $45 \mathrm{~mm} \times 25 \mathrm{~mm} \times 10 \mathrm{~mm}$ (see Fig. 2).

Due to limited neutron beam time, the number of specimens is fixed at three. However, in the neutron experiment, AG1 did not exhibit any significant water uptake. Therefore, only 
the results for VD4-1 and VD4-2 are presented in this paper for a detailed analysis of liquid water uptake.

\subsection{Cumulative Porosity Distribution from Microtomography}

The pore structures of the three specimens are imaged with the X-ray micro-computed tomography (micro-CT) setup of Empa. The setup consists of an X-ray source (X-ray tube "XT9225TEP”, Viscom), an XYZ linear stage (composed of three linear stages "LS-270", Micos) for positioning the specimen, a rotation table ("UPR-160 F air", Micos), and an X-ray detector ("XRD 1621 CN3ES", Perkin-Elmer). The X-ray tube was operated at $220 \mathrm{kV}$ and $0.1 \mathrm{~mA}$, and the emitted $\mathrm{X}$-ray spectrum was hardened with a 0.5 -mm-thick $\mathrm{Cu}$ filter. The focal spot size was below the nominal spatial resolution of $55 \mu \mathrm{m}$, which was calculated from the detector pixel size $(0.4 \mathrm{~mm}, 2 \times 2$ binned), the distance between the source and the object (158 $\mathrm{mm})$, and the distance between the source and the detector $(1146 \mathrm{~mm})$. The three-dimensional distribution image of the attenuation coefficient was reconstructed by means of a Feldkamp algorithm from 720 projection images $(1024 \times 800$ pixel, $3.6 \mathrm{~s}$ exposure $)$ recorded from different viewing angles distributed evenly over $2 \pi$ (Feldkamp et al. 1984).

In Fig. 2, the visible light photographs (a, f), the two-dimensional cumulative porosity distributions (CPD) obtained from reconstructions of 3D micro-CT scans (b, g), and the micro-CT slices at the front, middle, and rear positions of VD4-1 (c,d,e) and VD4-2 (h, i, j) are shown. The procedure to obtain the CPD from micro-CT images is as follows. All the 2D micro-CT images are binarized using an automatic threshold as calculated by the open-access image processing software Fiji (Schindelin et al. 2012). This process converts each pixel in an image to either a pore (pixel value $=1$ ) or a solid i.e. binder/aggregate (pixel value $=0$ ). The final CPD image is then obtained by adding all the images along the direction perpendicular to the image plane and then dividing the resulting image by the total number of images. The absence of porosity, i.e. an aggregate that crosses the entire thickness of the specimen, results in pixels with a value of 0 (white). All the other pixels have values ranging between 0 and 1 , where 1 (black) represents pixels where pores cross the entire thickness of the specimen.

The CPD was calculated from micro-CT images with two restrictions: voids smaller than $150 \mu \mathrm{m}$ were not detected due to resolution limitation, and the specimen was not a representative volume element (RVE) of the material as its thickness of $\approx 10 \mathrm{~mm}$ and the largest aggregate size $(11 \mathrm{~mm})$ were of similar dimensions. An ideal RVE should have dimensions which are larger than at least 3 times the aggregate size, but the available neutron beam does not have the energy to go through such a thick specimen. Hence, the porosity distribution calculated from the specimen is not a representative of the porosity of PA in the pavement scale, but is useful for the analysis of neutron radiography images.

\subsection{Experimental Setup and Procedure}

\subsubsection{Neutron Radiography}

The experiments were performed at the NEUtron Transmission RAdiography (NEUTRA) beam line of the Paul Scherrer Institute (PSI) in Villigen, Switzerland. The source of the neutron beam is the Swiss Neutron Spallation Source (SINQ), which provides a neutron beam in the thermal spectrum i.e., neutrons with a most probable energy of $0.025 \mathrm{eV}$ and a speed of $2200 \mathrm{~m} / \mathrm{s}$. Neutrons in the thermal energy spectrum have a high interaction with water, making the NEUTRA beamline appropriate for tracking the flow of water inside porous materials. The features of this facility include high collimation ratio, low gamma background, 

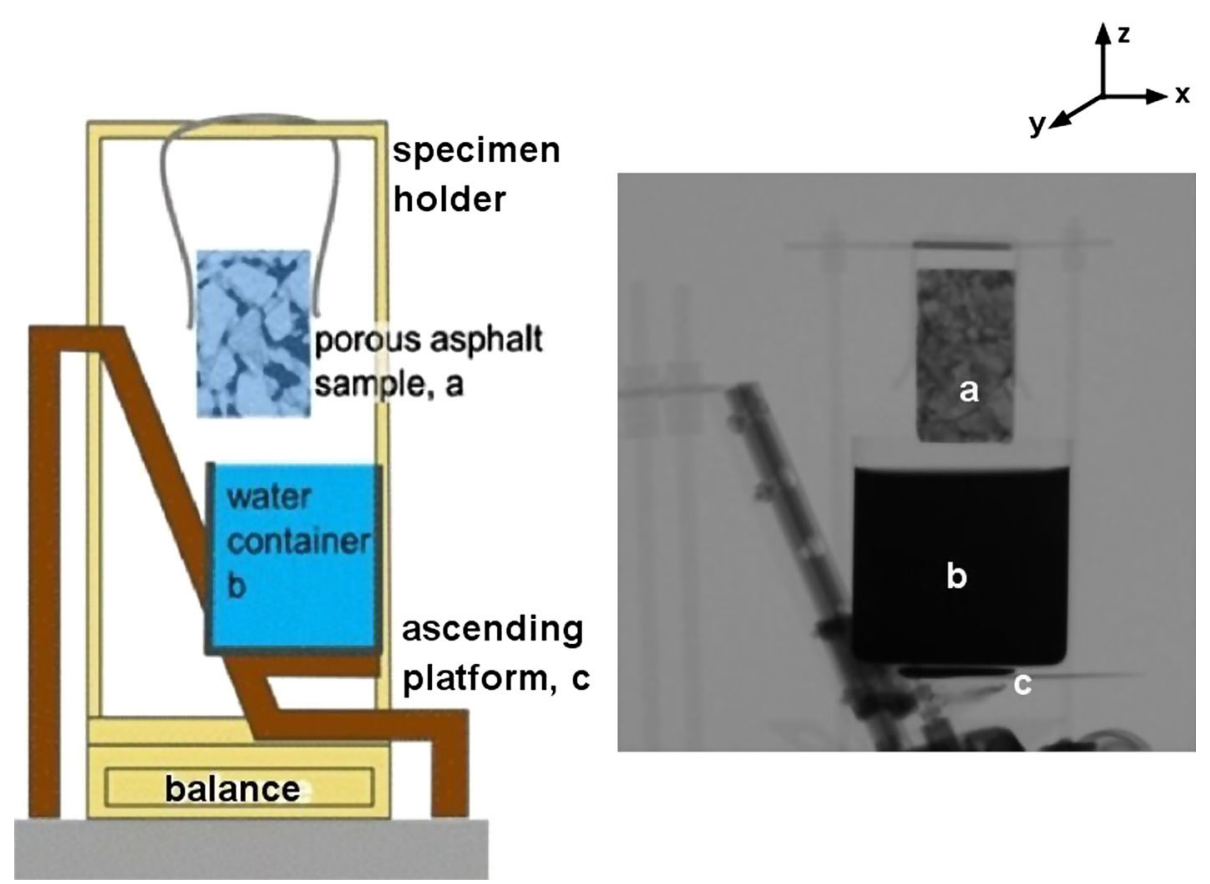

Fig. 3 Schematic representation of the experimental setup (left) and corresponding neutron radiograph image (right) showing: (a) PA specimen, (b) water container and $(c)$ ascending platform

and large beam diameter. A detailed description of the entire setup is given by Lehmann et al. (2001). The detector used is a scintillator-CCD camera-system, with a field-of-view of $152 \times 152 \mathrm{~mm}^{2}$. The camera used is Andor iKon-L with $2048 \times 2048$ pixels with a region of interest setting of $1505 \times 1505$ pixels, providing a field-of-view of $152 \times 152 \mathrm{~mm}^{2}$. The exposure time in the experiments was $12 \mathrm{~s}$ per radiograph with an attained spatial resolution of $100 \mu \mathrm{m}$ per pixel. The neutron chamber had a temperature of $22{ }^{\circ} \mathrm{C}$ and $30 \%$ relative humidity during the experiments.

\subsubsection{Uptake Experimental Setup and Procedure}

To image the PA specimens during the water uptake process, a custom-made setup was fabricated. It consisted of two parts: a specimen holder resting on a precision balance of $\pm 0.1 \mathrm{mg}$ accuracy and a remote-controlled ascending platform to raise the water container. Figure 3 shows a schematic of the experimental setup and a neutron radiograph of the same.

The specimens were initially dried in a convection oven at $55^{\circ} \mathrm{C}$ for longer than 10 days until they reached a constant mass, and were then stored in a desiccator at room temperature $\left(22{ }^{\circ} \mathrm{C}\right)$ until the time of the experiment when a reference image was acquired in dry state. Then, the specimen was coated with self-adhesive thin aluminum tapes on all the surfaces except the bottom $x-y$ plane (refer Fig. 2) and was installed in the specimen holder with the platform in low position, with no contact with water. Once the neutron beam was activated, the water container was elevated remotely until the specimen came into contact with water; this can be verified visually using neutron images (Fig. 3, right). The water uptake was from the bottom $x-y$ plane while the two-dimensional neutron radiographs capture the 
propagation of water in the $x-z$ plane. Radiographs were acquired initially every $12 \mathrm{~s}$ for the first 100 images and thereafter every 2 min for the duration of the experiments which lasted a minimum of three hours. The mass of the specimen was measured at the beginning and end of each experiment on a separate balance, as well as during the experiment with the setup balance at one minute intervals. These measurements are used as control values for the corrections of the radiographs, which are detailed in Sect. 2.3.4.

\subsubsection{Derivation of Water Content from Neutron Beam Attenuation}

Neutron radiography is based on the different attenuation levels undergone by a neutron beam as it traverses different objects, the attenuation being particularly high in water.

The Beer-Lambert law for the attenuation of radiation passing through matter can be written as

$$
I=I_{0} \mathrm{e}^{-(\Sigma / \rho) \rho d},
$$

where $I$ is the final beam intensity after the beam traverses a distance of $d$ in the specimen, $I_{0}$ is the incident beam intensity, $\rho$ is the mass density of the specimen, $\Sigma$ is the attenuation coefficient, and the term $(\Sigma / \rho)$ is the mass attenuation coefficient. From Eq. (1), we can calculate the mass thickness, $\tau(x, z)$, (Hassanein et al. 2005) i.e. the product of the specimen density and the specimen thickness at each pixel, as

$$
\tau(x, z)=\rho(x, z) d(x, z)=-\frac{\rho(x, z)}{\Sigma} \ln \frac{I(x, z)}{I_{0}(x, z)},
$$

where $(x, z)$ denotes the coordinates of a pixel.

In this study, the parameter of interest is the water content in each pixel at any time step, presented as mass per unit volume. As water moves through the material, any change of gray value of a pixel is attributed to the presence of water. Thus, the moisture content distribution $(\mathrm{MCD}), w_{t}(x, z)$, can be calculated as

$$
w_{t}(x, z)=\frac{\tau(x, z)_{t}-\tau(x, z)_{i}}{d_{y}},
$$

where $d_{y}$ is the constant specimen thickness in the direction of the beam ( $y$-direction), $\tau(x, z)_{i}$ and $\tau(x, z)_{t}$ are the initial mass thickness (of the dry specimen) and the mass thickness after a time $t$, respectively, for a given pixel. In terms of the beam intensities, Eq. (3) can be rewritten as

$$
w_{t}(x, z)=-\frac{\rho(x, z)}{\Sigma d_{y}} \ln \frac{I(x, z)_{t}}{I(x, z)_{i}}=-\frac{\rho(x, z)}{\Sigma d_{y}}\left[\ln I(x, z)_{t}-\ln I(x, z)_{i}\right],
$$

where $I(x, y)_{i}$ and $I(x, y)_{t}$ are the initial beam intensity (of the dry specimen) and the beam intensity after a time $t$, respectively, for a given pixel, as recorded by the detector after the beam traverses the entire thickness of the specimen. Since there is no deformation of PA during the experiments, the moisture content at each pixel can be calculated by simply subtracting the dry image from the wet images.

\subsubsection{Image Corrections}

To ensure an accurate quantitative determination of water content from the radiographs, the NR images have to be corrected for different types of artifacts. The corrections performed on the NR images in this experiment, in the order of their application, are as follows: 
1. Dark current correction: corrects the false signal from thermally generated electrons in the charge-coupled device of the CCD camera.

2. Black body correction: corrects the false signal from the neutrons which are scattered from the experimental setup or the environment to the detector.

3. Spectral effect correction: accounts for the shift in the mean neutron beam energy to higher energies after traversing a strongly attenuating specimen.

4. Intensity correction: scales the measured radiographs so that all the radiographs acquired in the experiment will have the same neutron source flux.

5. Flat-field correction: accounts for the spatial inhomogeneity of the neutron beam intensity or detector sensitivity.

When all the above corrections, except the spectral effect correction, are applied to the radiographs, Eq. (4) transforms to

$$
\begin{aligned}
w_{t}(x, z) & =-\frac{\rho(x, z)}{\Sigma z_{s}} \ln \frac{\{I(t) / I F\} / I(x, z)_{F F}}{\{I(i) / I F\} / I(x, z)_{F F}}, \\
I(t) & =I(x, z)_{t}-I(x, z)_{D C}-I(x, z)_{B B}, \\
I(i) & =I(x, z)_{i}-I(x, z)_{D C}-I(x, z)_{B B},
\end{aligned}
$$

where the subscripts $D C, B B$, and $F F$ refer to the dark current, black body, and flat-field correction reference images, respectively, while $I F$ is the Intensity Factor which is purely a function of the beam quality.

Eqs. (1)-(7) were derived based on the assumption that the incident neutron beam is monochromatic. But in reality, the energy distribution of the NEUTRA beam is similar to a Maxwell spectrum at $23.8 \mathrm{meV}$ (Hassanein et al. 2005). The spectral effect correction takes this factor into account and Eq. (1) can then be rewritten as

$$
\frac{I}{I_{0}}=\frac{\int_{0}^{+\infty} P(E) \cdot \varepsilon(E) \cdot e^{-(\Sigma(E) / \rho) \rho d} \mathrm{~d} E}{\int_{0}^{+\infty} P(E) \cdot \varepsilon(E) \mathrm{d} E},
$$

where $E$ is the energy of the incident neutrons, $\varepsilon(E)$ is a parameter to account for the energy sensitivity of the detector, and $P(E)$ is the Maxwell-Boltzmann probability density function which represents particle speeds (energy) in gases.

The corrections were performed with the Quantitative Neutron Imaging (QNI) method used previously by Pleinert et al. (1999), Hassanein et al. (2005, 2006). For a detailed description of the algorithm implemented in the QNI method, the reader is referred to Hassanein (2006).

\section{Results and Discussion}

We first track water propagation in terms of MCD, as defined by Eq. (3), with units of $\mathrm{kg} / \mathrm{m}^{3}$, where the dry image at time $t=0$ is subtracted from the wet image at the given time step. As already mentioned, the AG1 specimen showed very little uptake of water. Hence, we only present the results of the tests on VD4 specimens. Figures 4 and 5 show the spatial and temporal variations of MCD of the two VD4 specimens, at five selected time steps. The time steps are chosen with the aim of documenting the main event of water redistribution. The selected time steps also make it possible to focus on the initial stages of uptake, where the uptake rate is expected to be higher.

In Figs. 4 and 5, the top row of images (a-e) are contour plots of MCD. In order to focus more effectively on the movement of water within the specimens, a second series of images 
(a)

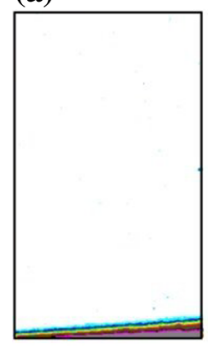

$1 \mathrm{~min}$

(f)

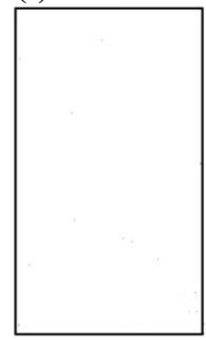

(b)

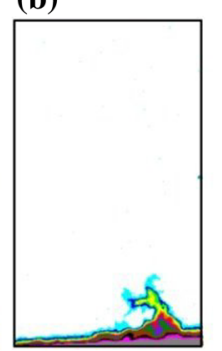

5 mins

(g)

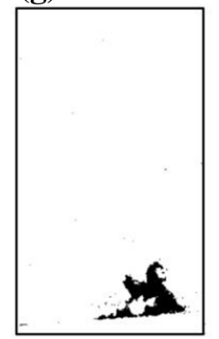

(c)

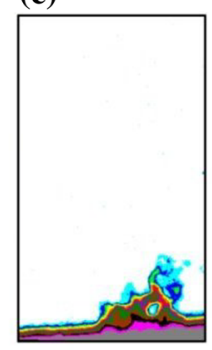

21 mins

(h)

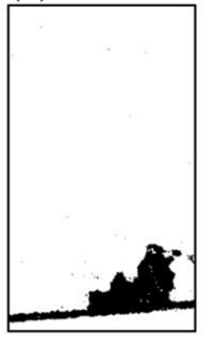

(d)

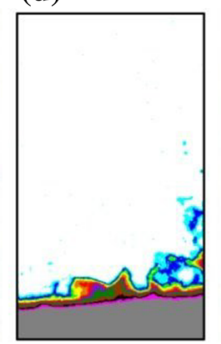

88 mins

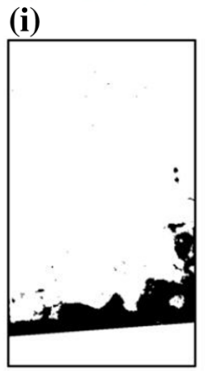

(e)

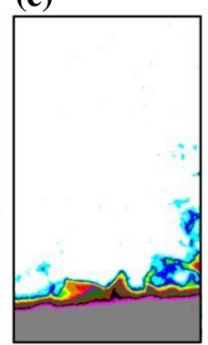

172 mins (j)

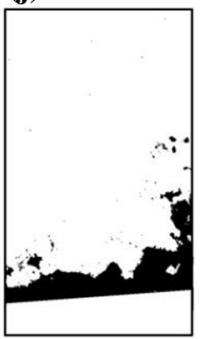

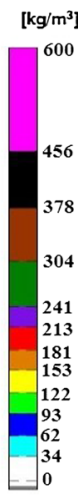

Fig. 4 (a-e) Temporal and spatial variations of the moisture content distribution (MCD) inside Specimen VD4-1 at five time steps. (f-j) MCD segmented at $30 \mathrm{~kg} / \mathrm{m}^{3}$ wet threshold, after subtraction from the wet image at time $=1 \mathrm{~min}$

(a)

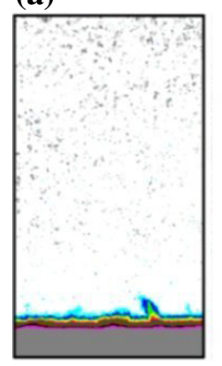

3 mins

(f)

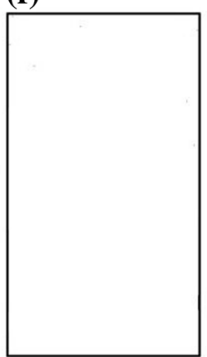

(b)

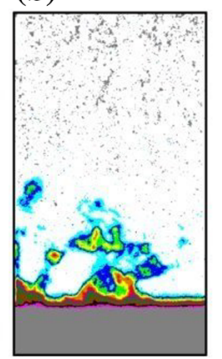

20 mins

(g)

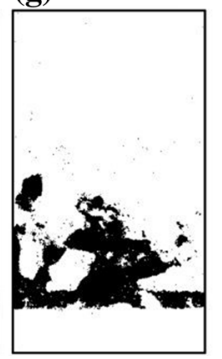

(c)

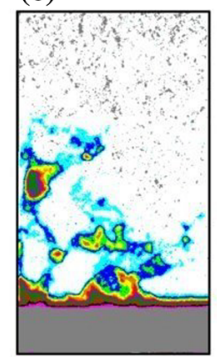

69 mins

(h)

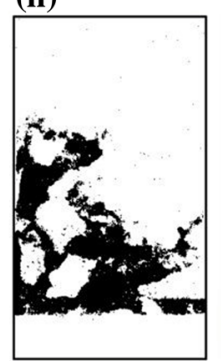

(d)

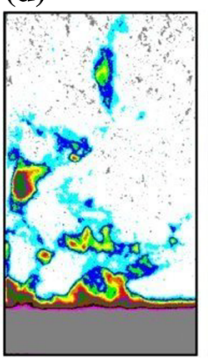

118 mins

(i)

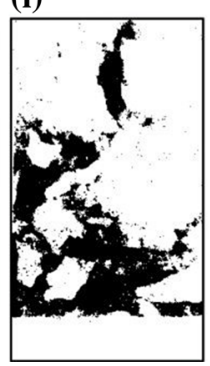

(e)

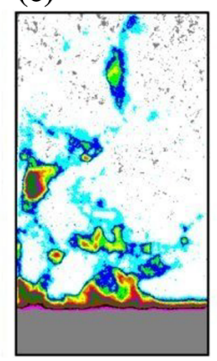

138 mins

(j)

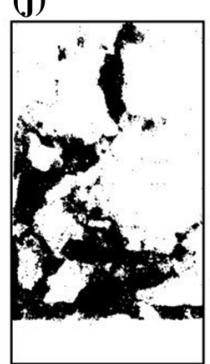

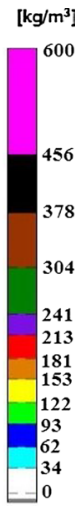

Fig. 5 (a-e) Temporal and spatial variations of the moisture content distribution (MCD) inside Specimen VD4-2 at five time steps. (f-j) MCD segmented at $30 \mathrm{~kg} / \mathrm{m}^{3}$ wet threshold, after subtraction from the wet image at time $=3 \mathrm{~min}$ 
(referred to as binarized images) are shown in the bottom row $(\mathrm{f}-\mathrm{j})$, where the first wet image in the upper row of images is subtracted from the wet images at the remaining time steps. In these images, pixels have a binary representation, white or black, where a black pixel represents a value that has crossed a threshold of $30 \mathrm{~kg} / \mathrm{m}^{3}$ when subtracted by the first wet image, while a white pixel represents a value that does not change (e.g., absence of porosity) or which is below the threshold. This threshold has been selected through trial and error to achieve a clear visualization of the water front, thereby indicating the areas in the specimen which undergo significant water uptake.

It is also to be noted that the levels of submergence of the bottom part of the specimens in water are different due to the different levels of ascension of the water container as this parameter was difficult to control remotely. Moreover, depending on the pore structure, different levels of ascension were necessary to initiate uptake. The exact level of submergence can be visualized by the gray pixels at the base of the specimens in the top row of images, and the white pixels at the base of the specimens in the bottom row of images. No analysis in these parts is done since they are submerged under water. Additionally, the white patches in between the wet areas indicate the areas of $0 \%$ porosity i.e., the areas where a single aggregate crosses the entire width of the specimen, as also visible in Fig. $2 \mathrm{~b}$ and $\mathrm{g}$ and Fig. 5e and $\mathrm{j}$.

We can make a few general observations from Figs. 4 and 5. It was seen that water travels a significant distance inside both VD specimens, with the higher uptake being in VD4-2. Furthermore, in both specimens, there is not much difference in MCD between the penultimate and final time steps. This implies that much before the final time step, all the paths which could potentially be followed by uptaken water have been filled and additional uptake of water had stopped. The exact time at which water uptake stops is influenced by a variety of factors like gravity, dead-end pores, the presence of pores with sharply diverging diameters etc. In VD4-1, the uptake initially begins on the right side of the specimen while in VD4-2, there is no clear preference. This aspect is investigated further later in this section. In VD4-2, in the last two time steps, we can see the emergence of a water island at the top part of the specimen. It can be further observed that, in VD4-2, there are high moisture content regions (yellow, green and red) surrounded by low moisture content regions (dark and light blue), where the low moisture content regions could be aggregate-dominated cross sections which are surrounded by high moisture content pore-dominated cross sections.

A more detailed analysis of water propagation is now studied in terms of another parameter, the degree of saturation (DoS), $s_{t}(x, z)$, defined as

$$
s_{t}(x, z)=\frac{w_{t}(x, z)}{\rho_{w} \cdot \phi(x, z)},
$$

where $\phi(x, z)$ is the two-dimensional porosity distribution as shown in Fig. 2, $w_{t}(x, z)$ is the MCD calculated with Eq. (3), and $\rho_{w}$ is the density of water.

Unlike the MCD analysis, the DoS analysis enables us to clearly identify those pores where saturated flow certainly occurs. For pores which show a DoS of less than $100 \%$, further analysis is required to distinguish between saturated and unsaturated flow, as described later in this section. At this stage, the flow in these pores will be referred to as (un)saturated flow. The transformation of MCD to DoS analysis is done for a Region of Interest (RoI) smaller than the specimen, as shown in Figs. 6 and 7 for specimens VD4-2 and VD4-1, respectively. The dimensions of the RoIs chosen for specimens VD4-2 and VD4-1 are $35 \mathrm{~mm} \times 25 \mathrm{~mm}$ and $9 \mathrm{~mm} \times 25 \mathrm{~mm}$, respectively. In addition to focusing on a RoI, the DoS analysis also focusses on a time interval of interest i.e., 80 and 13 min after the dry image is taken, for specimens VD4-2 and VD4-1, respectively. Thus, a time of 1 min means that it is a wet 


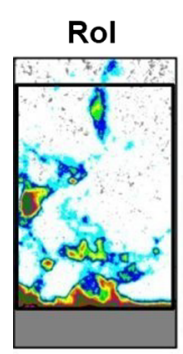

(a) $1 \mathrm{~min}$

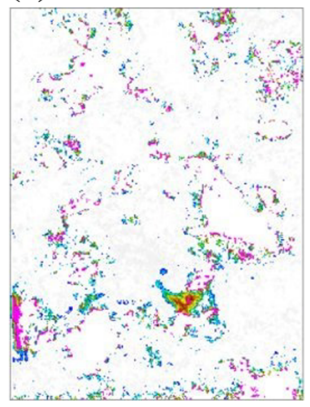

(d) 18 mins

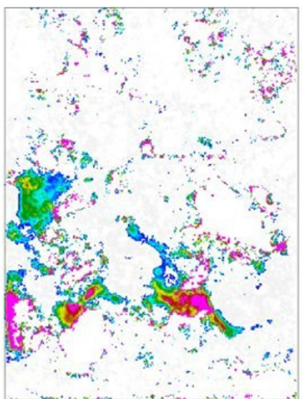

(b) 7 mins

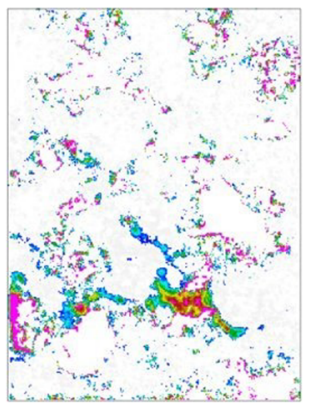

(e) 39 mins

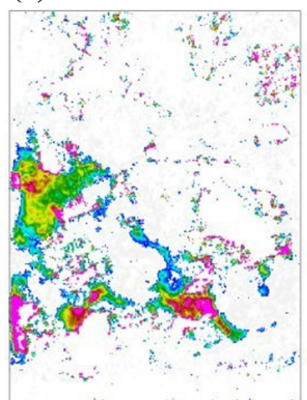

(c) 13 mins

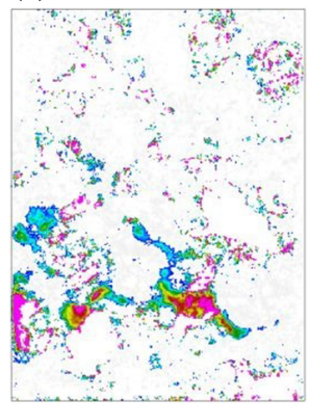

(f) $80 \mathrm{mins}$

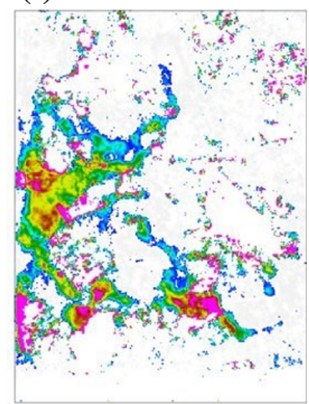

Fig. 6 Temporal and spatial variations of the degree of saturation inside the selected RoI of Specimen VD4-2
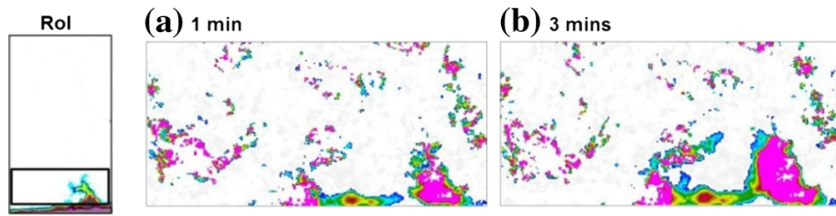

(c) 4 mins
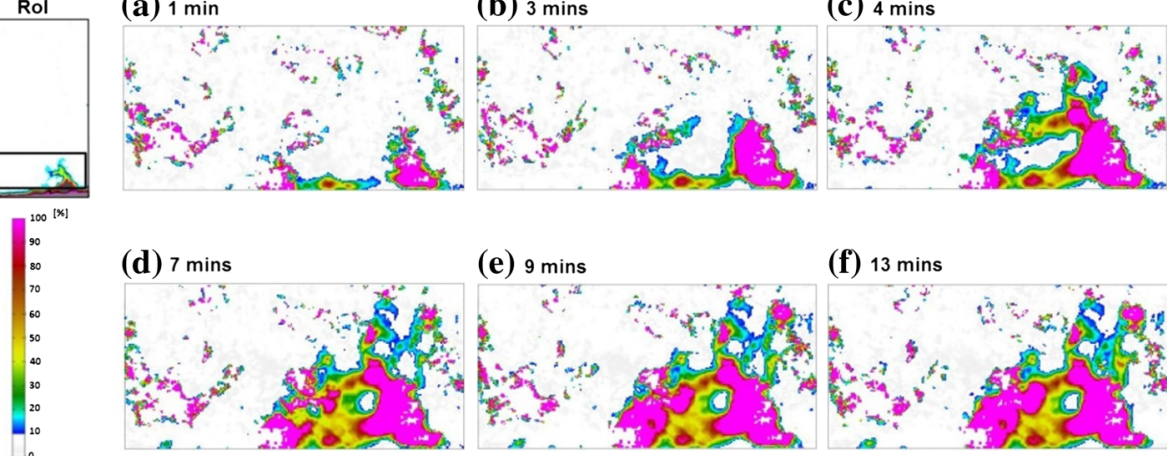

(f) $13 \mathrm{mins}$

Fig. 7 Temporal and spatial variations of the degree of saturation inside the selected RoI of Specimen VD4-1

image recorded 1 minute after the start of uptake, and so on. During the experiment, when water came in contact with the PA specimens using the ascending platform (see Fig. 3), the specimens moved laterally. Because of this movement, it was necessary to align the wet and dry images before they were subtracted. To mitigate the errors arising due to this registration process, the time steps in this analysis are chosen in such a way that during the experiment, there was minimum movement of the specimens between the dry image and the last wet image (i.e., 80 and 13 min for VD4-2 and VD4-1, respectively). Also, the levels of submergence of 
the specimens between these time steps are similar. As in the MCD images in Figs. 4 and 5, the large patches of white pixels in Figs. 6 and 7 indicate aggregates that run through the specimen, i.e. areas of zero porosity.

From Figs. 6 and 7, it can be observed that the patterns of water uptake are different in the two specimens. In VD4-2, the number of pores where saturated flow occurs is not large, as can be inferred from the low number of pink pixels at all time steps. However, as confirmed from Figs. 4 and 5, water travels much further in VD4-2 than it does in VD4-1, thereby indicating the importance of (un)saturated flows in VD4-2. Compared to VD4-2, VD4-1 has a larger number of pores where saturated flow is certainly active. In VD4-1, this number increases rapidly in the initial stages of water uptake (1, 3, 4 min). Toward the later stages $(7,9,13 \mathrm{~min})$, there is hardly any growth of this number.

We now focus on understanding more thoroughly the mechanisms by which water uptake takes place in PA. We pursue this next step using the VD4-1 results. To distinguish between saturated and unsaturated flow in the pores, we consider two datasets together: the data from the three-dimensional micro-CT scans and the two- dimensional DoS images. Since we are comparing $3 \mathrm{D}$ with $2 \mathrm{D}$ data, we have to restrict our region of interest to that part of the DoS images where the saturated and (un)saturated regions can be clearly distinguished. Moreover, the pore sizes as seen in the corresponding CT scans should be large enough to be able to neglect errors arising from the unavoidable imperfect overlapping of micro-CT and NR images. Hence, a smaller RoI is chosen within the RoI selected for Fig. 7. This RoI, which is shown in Fig. 8a, satisfies the above two conditions i.e., a clear pattern of uptake and large pore sizes. The dimensions of the RoI are also marked in Fig. 8a. To visualize the pore structure within this RoI, we have to study the evolution of the specimen cross section along the $z$ direction, with the help of micro-CT scans. This concept is illustrated in Fig. $8 \mathrm{~b}$ where the $x-y$ plane at a certain $z$ value is shown. These slices are of the dimension $25 \mathrm{~mm} \times 10 \mathrm{~mm}$. Figure 9 depicts the results of the approach described above. In Fig. 9a, the evolution of the specimen cross section within the RoI along the $z$ direction can be visualized with a series of micro-CT images at different heights. In Fig. 9b, the DoS distributions of the same RoI are shown at different time steps. These time steps are the same as used in Fig. 7. Positions along the $x$ direction are marked on all the figures. Additionally, the $z$ values of the DoS distribution in Fig. 9b, at which the corresponding micro-CT scans in the $x-y$ plane are shown in Fig. 9a, can be matched by the color code given for each $z$ value in Fig. 9a and its location in Fig. 9b.

In Fig. 9a, it can be observed that there are five large pores at the bottom of the specimen $(z=0.53 \mathrm{~mm})$, named pores A, B, C, D, and E, through which water can enter. Uptake of water initiates at the three positions marked $\mathrm{P}, \mathrm{Q}$, and $\mathrm{R}$ in Fig. 9a and b. The uptake at position $\mathrm{P}$ is due to pore $\mathrm{B}$, the uptake at position $\mathrm{Q}$ is due to pores $\mathrm{C}$ and $\mathrm{D}$ while the uptake at position $\mathrm{R}$ is mainly due to pore $\mathrm{D}$ and the connection between pores $\mathrm{D}$ and $\mathrm{E}$. The uptake at position $\mathrm{P}$ stops midway along the $z$-direction, whereas the uptake at position $\mathrm{R}$ is the strongest and appears to be saturated flow. At position Q, the uptake is mostly unsaturated and it also stops very early. With time, uptake seems to be active only at position $R$. The water uptaken at $R$ even flows laterally and fills up the pores above the points at which the uptake from positions $\mathrm{P}$ and $\mathrm{Q}$ stopped. This means that as expected, the uptake in PA is essentially a 3D process. However, due to the small thickness of the specimen, the complete development of this 3D flow remains limited.

Considering Fig. 9a and b, a few conclusions can be drawn. First, pore A does not play a significant role in water uptake. This could be due to the fact that it is a pore which is highly connected to the lateral boundaries or open to the surroundings, thereby incapable of any capillary uptake in saturated conditions. This is probably also true for Pore E. Pore 

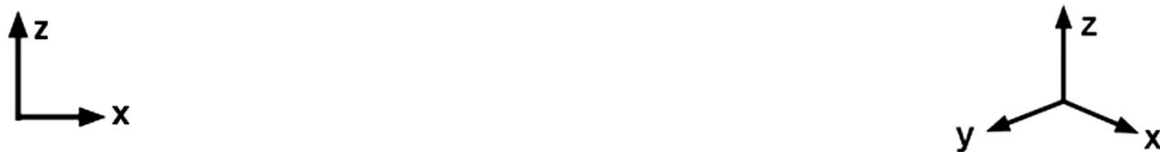

(a)

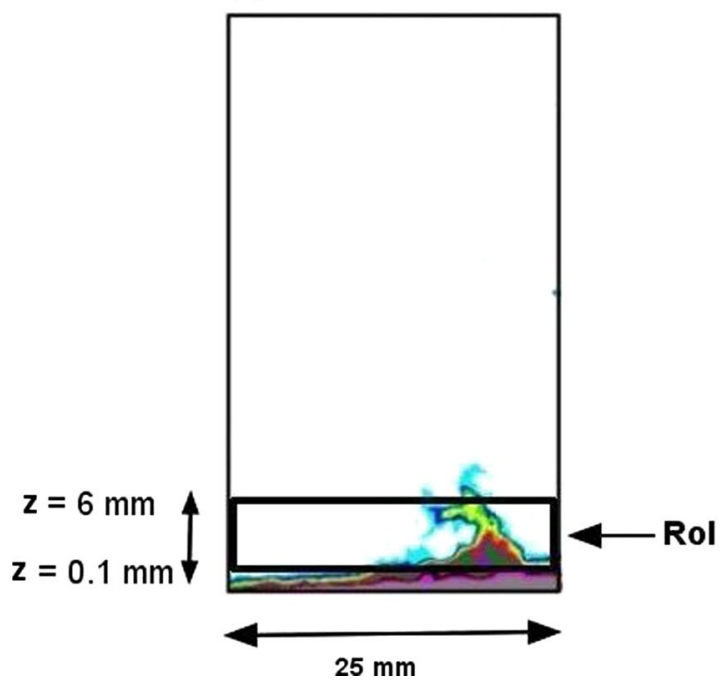

(b)

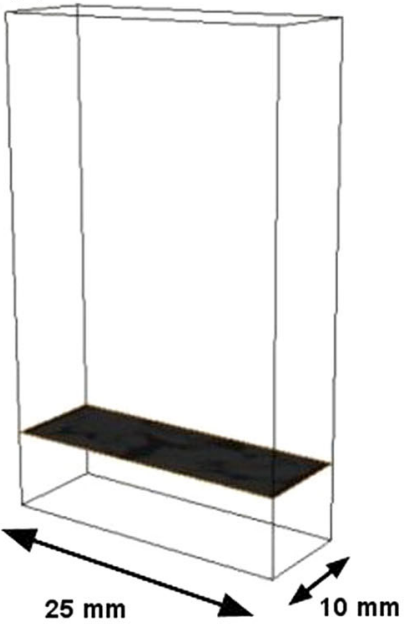

Fig. 8 For the analysis of the mechanism of water uptake, a an RoI is chosen from VD4-1 in which there is a clear pattern of uptake and $\mathbf{b}$ the three-dimensional structure of the selected RoIs is visualized with slices along the $z$ direction

C, though similar in topology to pores A and E, does exhibit some uptake at its left corner. Similarly, pore B also shows some uptake, but only at its right corner. Therefore, in these pores, unsaturated film/corner flow is expected to be the dominant mode of uptake. Going up the specimen along the $z$ direction, pore $\mathrm{B}$ gradually changes to a region with many binders-aggregate interfaces. This region is expected to be porous with very small holes, thereby allowing saturated flow through them. Due to this phenomenon, in combination with the film/corner flow in the left corner of pore $\mathrm{C}$, we can see a region of pink color between $x=10 \mathrm{~mm}$ and $x=15 \mathrm{~mm}$ from $t=7 \mathrm{~min}$ onward, around $z=1.96 \mathrm{~mm}$. Meanwhile, water uptake in pore $\mathrm{D}$ starts with film/corner flow on its right side. Going up the specimen, pore $\mathrm{D}$, which is initially not completely closed, reduces in size and splits into two well-defined pores $\mathrm{F}$ and $\mathrm{G}$, which are not connected to the surroundings. These two pores most likely display saturated flow as can be seen in the large pink-colored region between $x=15 \mathrm{~mm}$ and $x=20 \mathrm{~mm}$ from time $t=4 \mathrm{~min}$ onwards. The large yellow-colored region around $x=15 \mathrm{~mm}$ at this same time step is due to very little uptake in pore $\mathrm{C}$, which is open to the surroundings.

At $z=2.81 \mathrm{~mm}$, it can be seen that all the three pores (C, F, and G) merge into a single pore, but only for a short distance. At $z=3.92 \mathrm{~mm}$, the merged pores breakup into two new pores, pores $\mathrm{H}$ and $\mathrm{I}$. This merging and breakup seems to hinder the further saturated flow through the pores F and G. From the DoS distribution at $t=7 \mathrm{~min}$, it can be inferred that the new pores $\mathrm{H}$ and I now display film/corner flow at their corners, but definitely not saturated flow, because of the large yellow-colored region between $x=15 \mathrm{~mm}$ and $x=20 \mathrm{~mm}$. The presence of many small pores between $x=10 \mathrm{~mm}$ and $x=15 \mathrm{~mm}$ and around $x=20 \mathrm{~mm}$ leads afterward to fully saturated flows in these regions, as can be concluded from the large 


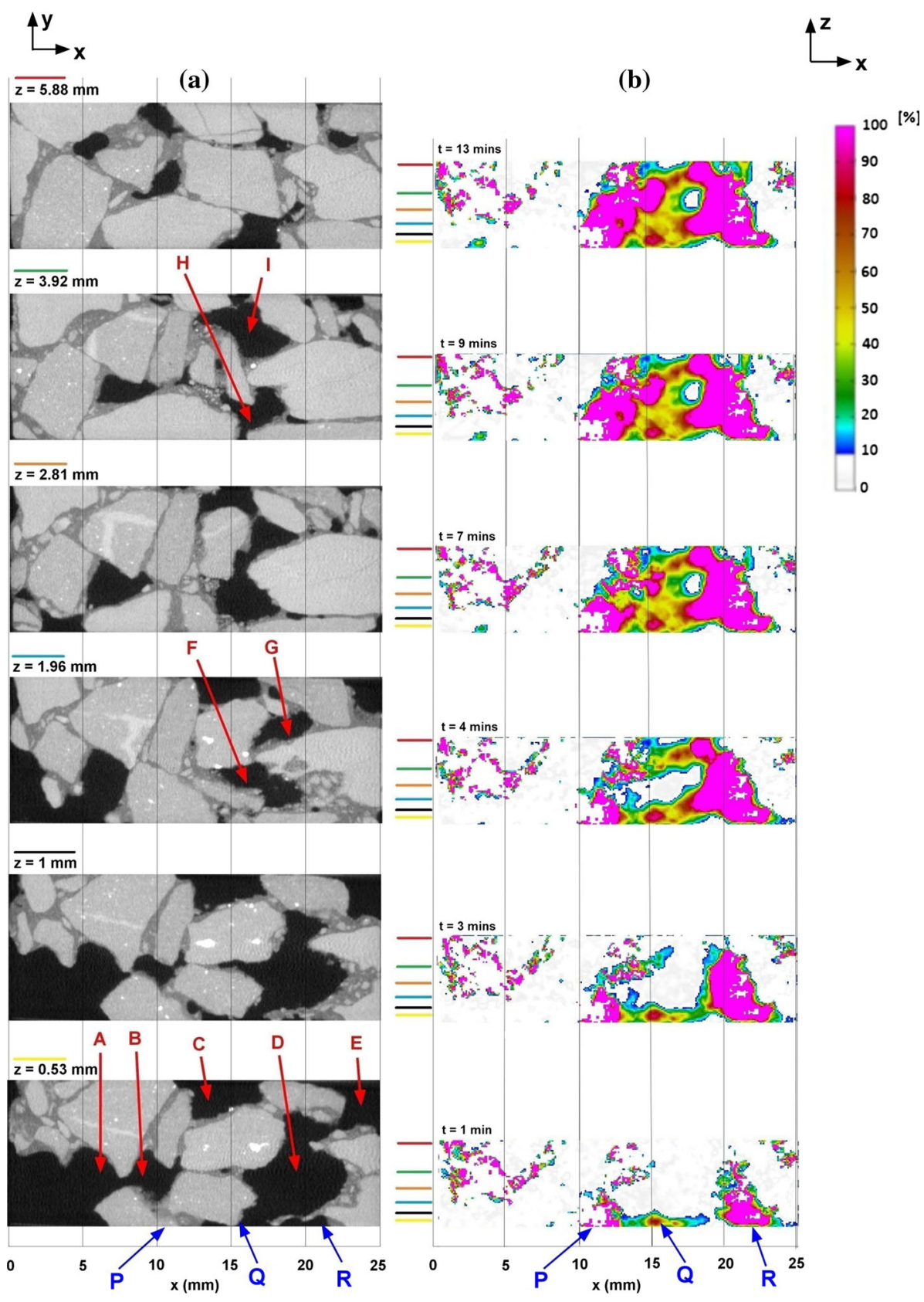

Fig. 9 Analysis of mechanism of water uptake in an RoI of VD4-1. a Micro-CT slices along the $z$ direction of the selected RoI and $\mathbf{b}$ DoS distribution of the RoI at different time steps. Large pores in the microstructure $(A-I)$ and the positions at which water uptake is initialized $(X-Z)$ are marked. The microstructure of the specimen at a certain $z$-value in (a) can be visualized by matching it with the section indicator of the same color in (b) 
pink-colored regions at these areas at $t=9$ and $13 \mathrm{~min}$. These pores are either fully within the mastic or in some cases surrounded partially by stone. Thus, these pores are generally smaller than the pores which are surrounded by large stones.

As water further moves upward with time, the region around $x=20 \mathrm{~mm}$ still has a significant amount of saturated flow due to the presence of many pores in the mastic, whose presence only increases along the specimen height. But saturated flow in the region around $x=15 \mathrm{~mm}$ reduces drastically since most of the pores in the mastic which were present at $z=3.92 \mathrm{~mm}$ are now replaced with impervious stone.

From this analysis, it is clear that there is a combination of saturated flow and unsaturated film/corner flow in the pores of PA despite the fact that the binder in the material is hydrophobic. This observation points to a complex interaction of water with the various components of PA, an aspect which needs to be further investigated in the future. It is also important to note that in an ideal pore surface, the binder covers all aggregates, leading to a hydrophobic surface throughout the pore system (Poulikakos and Partl 2012). However, in a real PA, not all surfaces are necessarily covered with binder as shown in previous microstructural investigations (Poulikakos and Partl 2010). This is especially the case for the VD4 specimens as they were in the field for seven years and thereby exposed to water, climate, and traffic. Therefore some of the binder from the aggregate surfaces could have been removed by stripping. This could very well be the reason why we did not observe any uptake in the laboratory-prepared un-aged AG1 specimen.

In a thin specimen, most of the pore network is open. Therefore, by using thicker specimens, we should be able to directly compare the magnitude of water uptake with pore connectivity, which is believed to be an important factor influencing water uptake. In other words, a thicker specimen would be desirable from the point of view of an ideal RVE. However, the inability of neutrons to penetrate very thick specimens limits the specimen thickness in NR experiments.

\section{Preferential Flow}

From the different figures presented in this study, it could be seen that water uptake in PA follows certain preferential paths. In other words, there is no occurrence of uniform waterfront as seen in isotropic microporous materials. Factors like tortuosity, pore accessibility, pore connectivity, and pore sizes play important roles in determining this preferential path. In case of VD4-1, which was in situ for 7 years, many physical and chemical factors will also play a role. In the following analysis, we limit our study to the influence of tortuosity and pore connectivity in determining the direction of the preferential path in VD4-1. For this purpose, the bottom part of the micro-CT scan of VD4-1 is divided into two RoIs, the right RoI (henceforth called RRoI) and the left RoI (henceforth called LRoI), as shown in Figs. 10 and 11. From Fig. 7, it is clear that, at the initial time steps, the RRoI has a higher water uptake than LRoI, i.e., water travels more easily in the RRoI. This characteristic makes the chosen RoIs ideal for the preferential flow analysis.

The software Avizo Fire 8.0 is used for the analysis presented in this section. First, a three-dimensional volume of the specimen is rendered from the micro-CT images. Then this volume is segmented with an automatic threshold into pores and non-pores (binders or aggregates). A fully saturated permeability simulation (i.e., absolute permeability) is then performed on this segmented three- dimensional volume. Absolute permeability is computed with a simplified Navier-Stokes equation system, with the following major assumptions: 


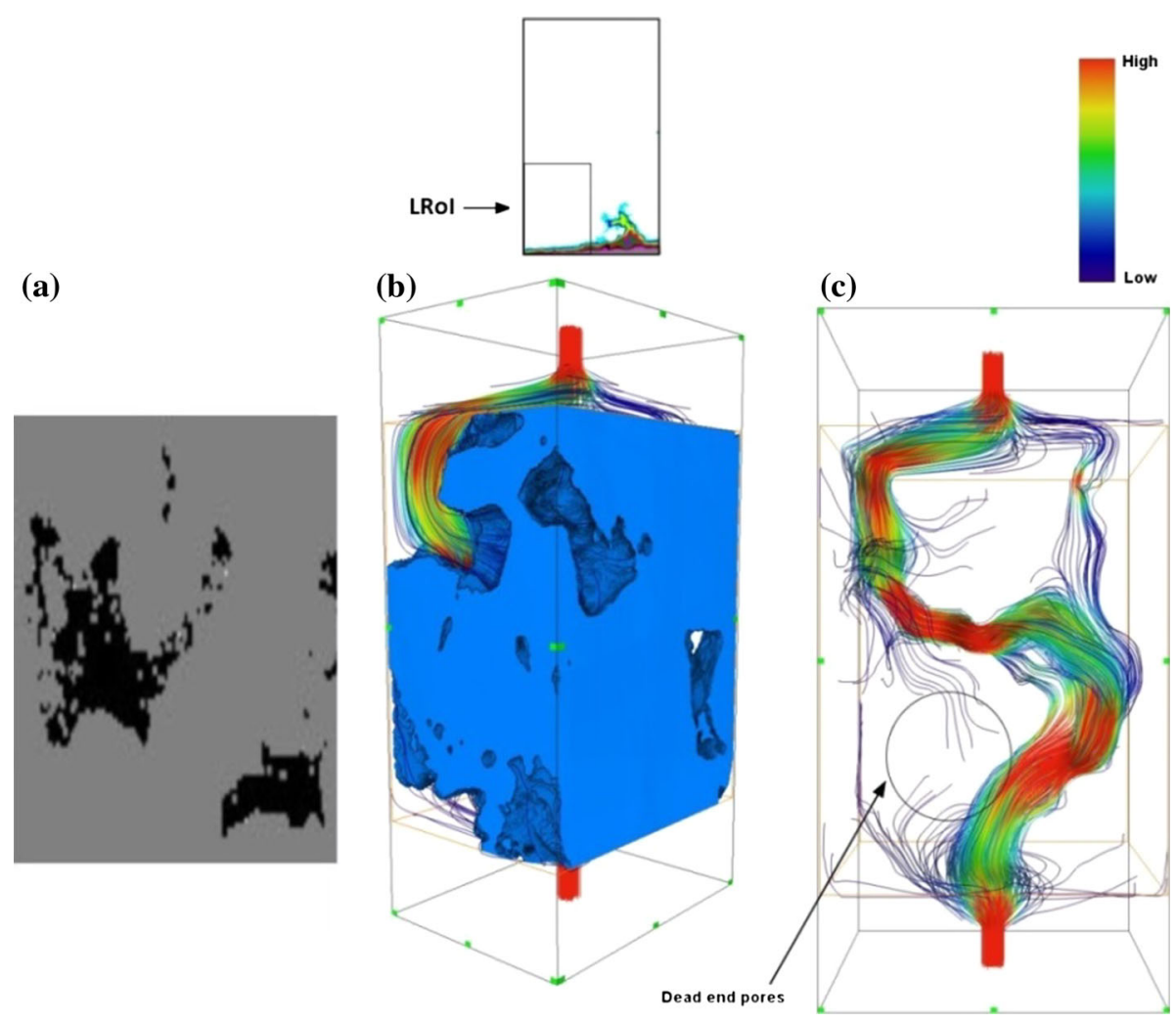

Fig. 10 Fluid path analysis of LRoI (Specimen VD4-1). a Binary porosity map, b 3D binary segmentation with a simulated fluid flow in the $z$ direction and $\mathbf{c}$ streamlines of the simulated fluid flow through the pore network, where the color map indicates the velocity of water

- the fluid is incompressible and Newtonian,

- the flow is steady-state and in the laminar regime.

A finite volume method with a staggered grid arrangement is used to solve the NavierStokes equations. The permeability experiment was simulated with a pressure difference of $30,000 \mathrm{~Pa}$ between the inlet and outlet, and the main direction of fluid flow was specified to be the $z$-direction (along the height of the specimen). The results from the simulations are presented in Fig. 10 (for LRoI) and 11 (for RRoI), along with an illustration of the selected RoIs.

Figures 10a and 11a are the binary porosity maps of the respective regions of interest. The black pixels represent regions with zero porosity while the gray pixels have a non-zero porosity value. The segmented volume combined with the streamlines of the simulated fluid flow is seen in Figs. $10 \mathrm{~b}$ and $11 \mathrm{~b}$. These figures provide a more graphical description of the simulated flow within the material structure. In Figs. 10c and 11c, for the sake of clarity, the predicted fluid paths are shown without displaying the three-dimensional material structure. In Fig. 10c, it can be clearly seen that there is one major path taken by the fluid, but that path is highly tortuous. Also, there are many paths which lead to dead ends. These dead-end pores, where the velocity of fluid falls to zero, are marked in Fig. 10c. From Fig. 10a, it can be seen that, in the LRoI, there are two clusters of non-porous pixels, one each on the 


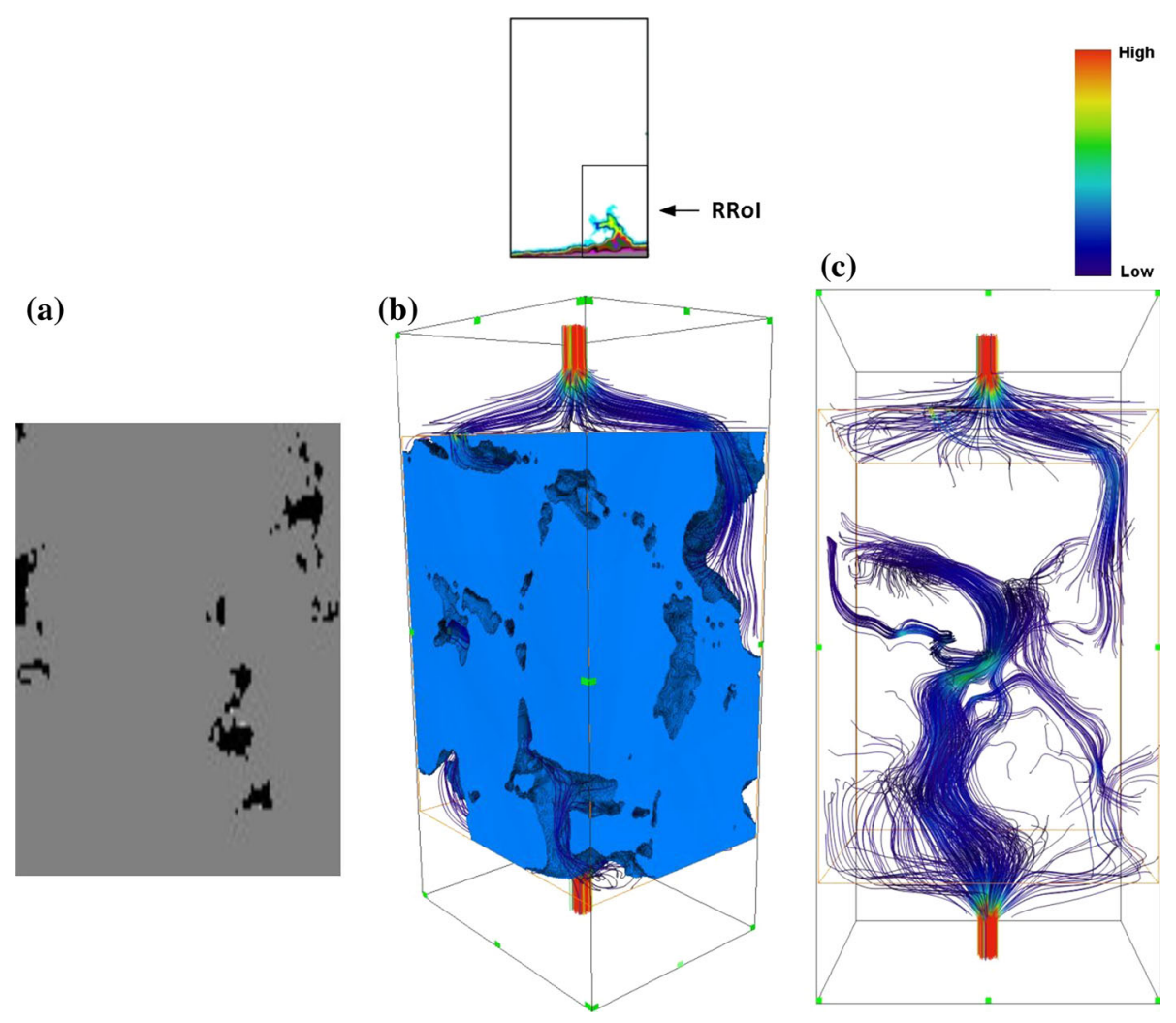

Fig. 11 Fluid path analysis of RRoI (Specimen VD4-1). a Binary porosity map, b 3D binary segmentation with a simulated fluid flow in the $z$ direction and $\mathbf{c}$ streamlines of the simulated fluid flow through the pore network, where the color map indicates the velocity of water

left and right sides. It is on the left cluster that most of the dead-end pore networks end. Because of the presence of these clusters, the water has to flow upward between them, which results in the highly tortuous path. Hence, water cannot travel upward easily in the LRoI. On the contrary, it can be seen in Fig. 11c that there are multiple paths available for water to travel in the RRoI, most of which are relatively less tortuous. Also, in Fig. 11a, no big clusters of non-porous pixels are present. Hence, the presence of relatively less tortuous paths with higher pore connectivity could be one of the most important reasons why water travels upward more easily in the RRoI, as compared to the LRoI.

\section{Conclusions}

Water uptake in PA was studied using NR on three different PA specimens. While the laboratory-prepared un-aged PA specimens hardly showed any uptake, we observed considerable uptake in the aged specimens taken from the road. MCD plots of the aged PA specimens were derived from the NR images, from which the paths followed by water became clearly visible. By combining NR and micro-computer tomography, saturation degree distributions were plotted, from which the regions where saturated flow was certainly active could be 
identified. In order to clearly distinguish between saturated flow and unsaturated flow in the other regions, the three-dimensional microstructure of the specimen was combined with its two-dimensional saturation degree distributions. It was observed that uptake began mainly as unsaturated film/corner flow in the large pores which were not too open to the surroundings. As this uptaken water traveled further into the material, we observed a combination of saturated flow and unsaturated film/corner flow. Saturated flow was active mostly in the small pores which were completely or partially surrounded by mastic. Thus, we observed a co-occurrence of both unsaturated and saturated flows in aged PA despite the fact that the binder in PA is hydrophobic. This was due to the exposure of the hydrophilic aggregates to water, which is in turn due to the stripping of binder from aggregate surfaces due to years of mechanical and environmental loads. Moreover, by simulating an absolute permeability experiment, we determined the roles played by tortuosity and connectivity of the pore system in determining the speed and direction of water uptake. It was found that the region with relatively less tortuous and more connected paths showed faster uptake during the experiments.

Acknowledgments The experiments were carried out at the NEUTRA beamline of the Paul Scherrer Institute, Villigen, Switzerland. The technical support of NEUTRA support team is greatly appreciated. The contributions and support of Stephan Carl and Markus Erb, technicians at EMPA, are gratefully acknowledged. This research was supported by a Swiss National Science Foundation (SNSF) Grant (200021_143651).

\section{References}

Cahill, T.H., Adams, M., Marm, C.: Asphalt: the right choice for porous pavements. Better Roads 74, 60-66 (2004)

Cnudde, V., Dierick, M., Vlassenbroeck, J., Masschaele, B., Lehmann, E., Jacobs, P., Van Hoorebeke, L.: High-speed neutron radiography for monitoring the water absorption by capillarity in porous materials. Nucl. Instrum. Methods Phys. Res. B 266, 155-163 (2008)

El Abd, A.E.G., Milczarek, J.J.: Neutron radiography study of water absorption in porous building materials: anomalous diffusion analysis. J. Phys. D 37, 2305-2313 (2004)

Feldkamp, L.A., Davis, L.C., Kress, J.W.: Practical cone-beam algorithm. J. Opt. Soc. Am. A 1, $612-619$ (1984)

Hassanein, R., Lehmann, E., Vontobel, P.: Methods of scattering corrections for quantitative neutron radiography. Nucl. Instrum. Methods Phys. Res. A 542, 353-360 (2005)

Hassanein, R., Meyer, H.O., Carminati, A., Estermann, M., Lehmann, E., Vontobel, P.: Investigation of water imbibition in porous stone by thermal neutron radiography. J. Phys. D 39, 4284-4291 (2006)

Hassanein, R.: Correction methods for the quantitative evaluation of thermal neutron tomography $\mathrm{PhD}$ Thesis ETH Zurich, Switzerland (2006)

Kim, H., Sokolov, K., Poulikakos, L.D., Partl, M.N.: Fatigue evaluation of carbon FRP-reinforced porous asphalt composite system using a model mobile load simulator. Transp. Res. Rec. 2116, 108-117 (2009)

Lehmann, E.H., Vontobel, P., Wiezel, L.: Properties of the radiography facility NEUTRA at SINQ and its potential for use as European reference facility. Nondestruct. Test. Eval. 16, 191-202 (2001)

Maupin, G.W.: Asphalt permeability testing: specimen preparation and testing variability. Transp. Res. Rec. 1767, 33-39 (2001)

Meiarashi, S., Ishida, M., Nakashiba, F., Niimi, H., Hasebe, M., Nakatsuji, T.: Improvement in the effect of drainage asphalt road surface on noise reduction. Appl. Acoust. 47, 189-204 (1996)

Partl, M.N., Canestrari, F., Grilli, A., Gubler, R.: Characterization of water sensitivity of asphalt mixtures with coaxial shear test. Road Mater. Pavement Des. 9, 247-270 (2008)

Pleinert, H., Körner, S., Lehmann, E.: Neutron signal transfer analysis. Nucl. Instrum. Methods Phys. Res. A 424, 177-182 (1999)

Poulikakos, L.D., Gubler, R., Partl, M., Pittet, M., Arnaud, L., Junod, A., Dumont, A., Simond, E.: Mechanical properties of porous asphalt, recommendations for standardization Eidgenössisches Departement für Umwelt. Verkehr, Energie und Kommunikation UVEK, Bundesamt für Strassen (2006)

Poulikakos, L.D., Partl, M.N.: Investigation of porous asphalt microstructure using optical and electron microscopy. J. Microsc. 240, 145-154 (2010) 
Poulikakos, L.D., Partl, M.N.: A multi-scale fundamental investigation of moisture induced deterioration of porous asphalt concrete. Constr. Build. Mater. 36, 1025-1035 (2012)

Poulikakos, L.D., Sedighi-Gilani, M., Derome, D., Jerjen, I., Vontobel, P.: Time resolved analysis of water drainage in porous asphalt concrete using neutron radiography. Appl. Radiat. Isot. 77, 5-13 (2013a)

Poulikakos, L.D., Saneinejad, S., Sedighi-Gilani, M., Jerjen, I., Lehmann, E., Derome, D.: Forced convective drying of wet porous asphalt imaged with neutron radiography. Adv. Eng. Mater. 15, 1136-1145 (2013b)

Satija, R., Jacobson, D.L., Arif, M., Werner, S.A.: In situ neutron imaging technique for evaluation of water management systems in operating PEM fuel cells. J. Power Sources 129, 238-245 (2004)

Schindelin, J., et al.: Fiji: an open-source platform for biological-image analysis. Nat. Mater. 9, 676-682 (2012)

Strobl, M., Manke, I., Kardjilov, N., Hilger, A., Dawson, M., Banhart, J.: Advances in neutron radiography and tomography. J. Phys. D 42, 243001-243021 (2009)

Tuller, M., Or, D.: Hydraulic conductivity of variably saturated porous media: film and corner flow in angular pore space. Water Resour. Res. 37, 1257-1276 (2001) 